\title{
SHARING IN A COMMON LIFE: PEOPLE WITH PROFOUND AND MULTIPLE LEARNING DIFFICULTIES
}

\section{INTRODUCTION}

There is a view that what we owe to other people is explained by the fact that they are human beings who share in a common human life. There are many ways of construing this explanatory idea, and I explore a few of these here; the aim is to look for constructions that contribute to an understanding of what we owe to people with profound and multiple learning difficulties and disabilities (PMLD). I suggest that there are such constructions, and that these are illuminating for their emphasis on the social nature of human beings and on how we should take account of their potential. But equally, the contribution to explaining what we owe to people with PMLD has its limits: not every person with PMLD shares in a common human life, and the explanation of what we owe to those who do share in this life does not come to an end merely because it is established that this is what they do.

I will not say much about how we should understand the phrase 'what we owe to other people', nor about its place in an account of human moral status, and I will sometimes simply refer to someone's moral status as shorthand for what we owe to that person. I take this as one largely unspecified focal point, because it is helpful to assume, with Scanlon, that the domain in which we owe something to another person is broader than justice, which is especially concerned with social institutions, and broader also than that part of morality taken up with obligations, which specify requirements largely arising from specific actions and undertakings (Scanlon 2000: $6-7) \cdot{ }^{1}$

In exploring the idea of sharing in a common life I construe 'sharing' as 'participating in', and 'common life' as the social life characteristic of the environment that

\footnotetext{
1 There are other ways of talking about the moral status of people with PMLD, which might instead emphasise their entitlements (Nussbaum 2006) or our duties towards them (Gunnarson 2008), but these are not discussed here.
} 
someone lives in. These construals will raise heckles and I discuss some objections as the argument proceeds. My principal purpose is to render the idea of sharing in a common life in terms that help explain its eligibility as a ground for establishing the moral status of people with PMLD. I do not attempt to demonstrate that participation is indeed status conferring, nor what this moral status exactly amounts to.

In section 2 I include some anecdotal evidence about people with PMLD. Evidence of this kind can only do so much, and it is introduced principally to illustrate the detail and range of capacities of people with PMLD, which can be lost from view under a general acronym or a stipulative definition. Much of the argument is reserved for the sections that follow, where, as throughout, I am concerned to respond to points raised by Wasserman and McMahan on the subject of assisted participation (Wasserman and McMahan 2012). These writers identify significant difficulties with the position adopted here, and I cannot claim to overcome all of them. Nevertheless, I will suggest that the scope for participation by people with PMLD is more than they might be prepared to allow.

The participatory options I examine each make some call on agency, if only as something hoped for in the future, including when hope flies in the face of expectation. Accordingly I look at conceptions of actual and potential participation in social life (sections 2 and 3), and at the idea of treating people as if they have the potential to participate, even when the existence of any such potential is unlikely (section 4). I conclude with some thoughts on the relation between participation and the moral status of profoundly disabled people, and about how much, and how little, the argument has achieved.

\section{ACTUAL PARTICIPATION}

Why is participation in a common life thought to be related to what we owe to other people? Participation may give evidence of rationality or critical reflection on one's own good; but for Kittay this matters less than a capacity for empathy, kindness and fellow feeling. Her profoundly disabled daughter belongs to the human moral community because: 
she has the capacity to enjoy her life, to share her joy through her smiles and laugher, to embrace those who show her love and care, and to bring joy to all those whose lives she touches - an individual who, through her warmth, her serene and harmonious spirit, and her infectious love of life enriches the lives of others .. (Kittay 2005: 123).

\section{Mulhall argues:}

We do not strive (when we do strive) to treat human infants and children, the senile and the severely disabled as fully human because we mistakenly attribute capacities to them that they lack ... We do it (when we do) because they are our fellow beings, embodied creatures who will come to share, or have already shared, in our common life, or whose inability to do so is a result of the shocks and ills to which all human flesh and blood is heir (Mulhall 2002: 18).

My interest is not in species-specific conceptions of misfortune, but in the argument from participation; and in particular the claim that what is owed to others is related either to their participating in forms of embodied common life open to human beings, or to there being the potential for such participation.

About actual participation Wasserman and McMahan argue:

If actual participation, however minimal, were required for moral status, many or most human beings with radical cognitive limitations would lack that status, because few have the skilled, concerted assistance that could make even that minimal participation possible for them - if anything could (Wasserman and McMahan 2012: 329)

The premise of this argument reflects a common belief. However, cognitive impairments are not all equally radical or profound. The Diagnostic and Statistical Manual of Mental Disorder (DSM-IV-TR) describes profound disability as including 'considerable impairment in sensorimotor functioning', 'retardation with some neurological condition' and as requiring the 'constant need for 'pervasive support' (DSM-IV-TR: 43-44.). In the fifth edition of the DSM profound disability in the social domain is such that 'the individual has very limited understanding of symbolic communication . . express[ing]. . desires and emotions largely through nonverbal, non-symbolic communication'. And in the practical domain the 'individual is dependent on others for all aspects of daily physical care, health and safety' (DSM-V 2013: 58; 61). 
Within the category of profound disability the variety of impairments is such that the Wasserman/McMahan claim is true for some children and false for many more others than their claim implies. Some persons will give evidence of the capacity for rationality, language use and reciprocity in personal relations. These people operate at the higher end of the P scale spectrum, as educationalists in England would say, whilst other children function at the lowest end of this spectrum. ${ }^{2}$ To give some idea of the range of capacities amongst children with PMLD we can contrast the abilities of children operating at the lowest and highest $P$ scales, as illustrated in the context of what is expected of children who follow an Art and Design curriculum. At the lowest level, P1(i) '[p]upils . . may be passive or resistant. They may show simple reflex responses, for example, startling at sudden noises or movements. Any participation is fully prompted' (QCA 2009: 4). Whilst at the highest level, P8:

Pupils develop their ideas and use materials .. working in two and three dimensions. They finish a piece of work following an established pattern of activity, for example, gathering appropriate materials, taking part in an activity and stopping work when finished.. They use a growing art vocabulary . . (London: Qualifications and Curriculum Authority 2009: 4-5)

One and the same pupil may be capable of subtle reciprocity in personal relations whilst not able to utter a single word; or she is able to notice and laugh at the incongruity evident in using a large broom as a paint brush, but cannot be left alone for a moment. Other persons will possess a more restricted range of capacities, and others, still, exhibit only slight evidence, if that, of any of the capacities associated with personhood.

Evidence for these claims is found in innumerable case studies (Dee et al. 2002), reports from the Inspectorate in England and Wales (Ofsted 2013), and in the detail of the $\mathrm{P}$ scales themselves, which state what is expected by experts in curricula and assessment across almost the entire cohort of children with PMLD (QCA 2009). Evidence of these kinds is not, of course, anything like conclusive: the expectations and beliefs of experts may be misguided or false. But in view of the extent of

\footnotetext{
${ }^{2} \mathrm{P}$ scales were created in response to a need to assess children who did not attain at least level 1 on the National Curriculum; see Njaji and Tymms 2009.
} 
consensus amongst those charged with assessing the capacities of profoundly disabled children it is evidence that has to be reckoned with.

At the same time, any capacity to participate is typically dependent on the extensive assistance of others, raising the question whether the act of participation can be attributed to the assisted person. If I have no muscle tone in my limbs and you move my arm we may ask if the act of moving my arm is mine. I remain the author of an act, even though it is not my action, if I ask you to move my arm, or you move my arm in accordance with my expressed preference. But if I cannot issue instructions or understand what you are doing when you move my arm then even this much is doubtful. These questions, about agency and authorship, are discussed in the context of assisted surrogacy (Silvers and Francis 2009) and they apply equally to any discussion about the participative acts of assisted persons.

Where agency and authorship are both in doubt, so too is participation. However, it is far from typical that someone with PMLD is neither agent nor author of her actions, nor therefore, that she does not participate in the social activity in which she is in some way involved. To substantiate this claim it is necessary to look further at what is required of a claim to participation. Wasserman and McMahan argue:

\begin{abstract}
The agency of a surrogate acting on behalf of a cognitively limited individual cannot be attributed to that individual unless she guides or endorses it. But an individual with radical cognitive limitations - who cannot see herself as a temporally extended being or engage in simple practical reasoning - cannot guide or endorse her surrogate's decisions or actions, so it is unclear how those decisions or actions can be regarded as hers.. Participating as someone's surrogate in social activities is not enabling that individual to participate if she cannot contribute to, direct, or endorse those activities; if she cannot understand the nature of those activities or her roles in them (Wasserman and McMahan: 331).
\end{abstract}

'Radical cognitive limitations' is here given a stipulative definition that does not apply to anything like the entire cohort of people with PMLD, as this category is understood, at any rate, in England. ${ }^{3}$ But this can be put to one side. A person is

\footnotetext{
${ }^{3}$ McMahan has his own reasons for choosing to define 'radical cognitive impairment' in the terms he prefers. But other definitions also have their uses: the category of PMLD, referred to here, allows for large differences between the highest and lowest functioning human beings included in this cohort, as indicated by the varying demands at the higher and lower end of the P scales.
} 
said to participate in social activities only if she understands their nature, and 'guides', 'contributes to', 'endorses' or 'directs' those activities. I discuss these varying options in the course of defending the claim that at least most children with PMLD have a capacity to participate in the social life of their family home, school or neighbourhood.

Murilo is a six year old Brazilian boy, who has a diagnosis of global developmental delay and severe epilepsy - he tends to have more than 70 seizures daily. Nevertheless, according to his teacher, Karian Schuitema, Murilo is a sociable boy who likes to interact with others; although he has no more than a handful of words he communicates with familiar adults by pointing, signing, vocalising and by means of facial expressions. At the time of observation (by Schuitema) Murilo is about to return home from school and he and Schuitema are waiting for his transport to arrive:

Karian kneels down in front of the school gate and Murilo sits on her leg. He is pre-occupied at first but when he notices that Karian is looking at him, he raises his finger slightly. Karian immediately recognizes what he wants: they have perfected 'their' joke since he first joined the class one and a half years ago. It began when Murilo would do something not allowed - swiping a cup of water off the table for example - and he would want the adults to wag their finger whilst exclaiming 'no, no, no!'. As time went by Karian adapted this practice so that she got into the habit of saying 'la, la, la', whilst wagging her finger, and doing this not in response to any mis-behaviour but as part of a playful routine. It has since become such a common practice that the first thing Murilo now does when he sees Karian is to raise his finger so as to prompt a 'la, la, la' and a wagging of the finger, and this game is repeated throughout the day (Schuitema et al, forthcoming).

This is one of innumerable examples of how children with PMLD interact with others, and it illustrates that a child who is by no means operating at the highest $P$ levels is able to participate in social activity. 'Participation' here takes the form of contributing to a playful routine that has become a familiar feature of daily interaction. Murilo does not direct the exchange, although he can initiate it, by raising his finger, and he can guide it in so far as he indicates when he wishes to begin, how long he wishes to keep it up and when he has had enough. And he clearly understands at least this, that by raising his finger he is initiating an exchange that Schuitema is expected to contribute to, with subsequent contributions which together amount to a routine that is intended by each as playful and funny. 
A person can participate in social life in virtue of what he brings to relationships, or as having a character that impresses itself upon a community, or by acquiring a social role in virtue of becoming known for a sense of mischaeviousness. Murilo contributes to the character and pattern of social inter-action with Schuitema, and with his peers and teachers, although he will not recognise his contribution in the terms used here.

This last claim warrants more attention. Tim Webb is the Director of Oily Cart, a theatre company specialising in the production of interactive theatre for children with profound learning difficulties. 'Communion' and 'participation' are two aspects of the theatre he aspires to create:

Theatre is about communion with one another. That is a difficult thing to do and also a wonderful thing to do, despite all the barriers, disabilities, autistic tendencies . . . Then to break through that is wonderful, and you can think that anything is possible . . We learn so much from the children, we get so much inspiration from them . . . Theatre is a part of community - the whole community participates in theatre - in its magic, drama, beauty. When theatre is at its best everyone participates in it (interview with BLANK, in BLANK).

A person can understand her actions under numerous descriptions. She may not understand that she is taking part in interactive theatre, nor that she is contributing to a multi-sensory spectacle before an invited audience. But she may understand that she is waving coloured sticks and laughing in response to a clown whose trousers are falling down. And she may also anticipate what is asked of her, show willing compliance, and indicate by her vocalisation that she is intentionally responding, as intended, to the actors' communicative behaviour. It is not necessary that the children of Oily Cart should understand what they do in terms employed by a competent English speaker; what is required is that they should have some understanding of what they are doing, including as part of social interaction, and this does not in turn require that they should understand the nature of what it is that they are contributing to. Likewise, a group of infants may not recognise their actions in such terms as 'charging about' and 'shouting at the top of their voices', nor that that they are contributing to the pandemonium and high spirited melee of a birthday party. But if they understand that they are playing and laughing along with each 
other, they are participating in social activity nevertheless - in this case, a party that is threatening to get out of hand.

Of course, as I have acknowledged, the children Oily Cart work with cannot produce theatre by themselves; there is probably nothing that they could do unassisted that would contribute to this spectacle. What, then, of the claim that 'even if some radically impaired human beings had moral status on [the] basis [of participation], they would have it only contingently - the necessary assistance could be lost or withdrawn at any time' (Wasserman and McMahan 2012: 329)

Assume that the moral status attaching to participation is contingent if and when any capacity to participate is contingent. If by contingent we mean 'dependent on the assistance of others' we must explain why only people with profound impairments are dependent on others in a sense that renders their moral status more precarious than others. Of course, many people are less dependent on others than are profoundly impaired people, in the sense that there is a far greater range of actions that they are able to complete without assistance. But even so, in order to undertake many of their actions, including their unassisted actions, they are dependent on the past and present actions of other people.

When I present this paper at a seminar 50 miles from home I am dependent on school staff to look after my children. If staff do not look after my children I cannot attend the seminar; in this sense my participation in that event, and anything I do in the course of it, is contingent on the actions of other people. In other words, my participation causally presupposes the actions of other people in taking care of my children - and, for that matter, in getting the trains to run on time. If these are not contingencies that undermine moral status, then we must explain what distinguishes contingencies of this kind from the contingencies that attach to the acts of people with profound impairments. ${ }^{4}$

We can distinguish between actions which causally presuppose the past actions of others, and actions which require the contemporaneous assistance of others. My

\footnotetext{
${ }^{4}$ For the significance of the causal presuppositions of action for agency, see Graham 2002.
} 
attendance at a seminar requires that the train driver kept to his schedule, but the reading of my paper is something I can do alone. This is different from many of the actions of a profoundly disabled person, which both presuppose the past actions of others and their contemporaneous assistance. Even here, however, it is worth noticing that what we take as an example of unassisted action - reading a paper at a seminar - presupposes the actions of other people; I could not read this paper if others were not here to listen, or if there was no room set aside for the seminar. Nevertheless, it is plainly true that people with profound learning difficulties require far more assistance, past and contemporaneous, than many other people, even to stay alive, let alone to engage in the social life of a community. In this sense their capacity to participate is more dependent on the assistance of other people than it is for most people without cognitive impairments.

Even so, whilst the children of Oily Cart could not participate were it not for the real time assistance of other people, they do, nevertheless, participate. Why is any moral status associated with participation undermined because someone could not have participated without the ongoing assistance of others? The obvious answer is: if and when assistance is withdrawn, and participation becomes impossible, the participatory ground of moral status ceases to apply. If moral status derives from participation, assisted or otherwise, and participation is not an option, then any claim to moral status has to be given up. In order to deal with this objection, it is necessary to explore the significance of the fact that someone may have the potential for participation although she is unable to participate, including when assistance is forthcoming.

\section{POTENTIAL PARTICIPATION}

There is a distinction between the capacity to do something, and the potential to develop a capacity. A healthy two year old boy has the capacity to talk; a healthy fetus has only the potential to develop this capacity. The infant boy has the capacity to communicate only if the neurological and physiological structures are present causally to support the act of communication; he has the potential to develop this capacity only if there is some prospect that these structures will emerge at some 
future point in his life (cf. McMahan 2005: 329). On this account, an anencephalic infant has neither the capacity for thought, nor the potential to develop this capacity. ${ }^{5}$

Two kinds of case illustrate the potential to participate on the part of people with PMLD: in the first, a trustee or surrogate acts as an intermediary between someone with PMLD and other persons or institutions. In the second case, a person with PMLD has the capacity to participate if she receives the skilled assistance of a parent, carer, or teacher. I will discuss the second of these. ${ }^{6}$

Wasserman and McMahan identity three problems with a claim about the moral status of people with PMLD that depends on the potential for participation: (i) the potential for responsiveness is limited to 'human beings with the psychological capacity for some degree of mutual acknowledgement and reciprocity'; (ii) the potential will be realised only if a person with PMLD has the opportunity for sustained interaction with other human beings; and (iii), the same potential might be found in non-human animals exposed to extensive interaction with humans (op.cit.: 330 ).

About (i): there are, indeed, hard questions to be asked about the moral status of a human being who has no capacity for reciprocity, and no potential to develop this capacity, no matter how extensive the pedagogic and medical assistance. If there is no possibility of developing any capacity to participate then the claim on behalf of a non-responsive human being is likely to come down to a claim about the importance of species membership, or of someone being our fellow creature. There may be a good argument to be made along one or other of these lines but I will not be making that here. What should be said is that, in view of the history of ignorance, error and under-estimation in respect of the capacities of people with disabilities, and in light of advances in pedagogy and technology, we are entitled to insist on stringent criteria for assessment before reaching a conclusion that someone has no potential to develop the capacity for responsiveness.

\footnotetext{
${ }^{5}$ See DiSilvestro 2010 for an ingenious alternative view.

${ }^{6}$ The first kind of case is no less important, but questions of trusteeship and surrogacy raise issues beyond the scope of this paper.
} 
I have previously begun to discuss claim (ii), that the potential to participate will be realised only if a person with PMLD has the opportunity for sustained interaction with other human beings. This can be considered alongside claim (iii), that the potential to participate, in any sense that confirms human moral status, neither extends to all human beings nor excludes all non-human animals.

Claim (iii) has been emphatically denied: if we properly attend to the concept of 'human being', and the fundamental practices distinctive of human forms of life, we should see that there are some acts, participative and otherwise, that could only be attributed to human beings, and which it would be nonsensical to apply to other animals. When Diamond reminds us that 'we do not eat our dead', she means to identify a practice that distinguishes human from non-human life, and for reasons that emerge from a conceptual understanding of what it is to be a human being, and to lead and take part in a human life (Diamond 1978). This is a characteristically Wittgensteinian line of thought, and Diamond is not alone in pursuing it (see, for example, Gaita 2000). Whatever the merits of this orientation, however, it is not one to be adopted here; rather, I will argue that we can point to examples of behaviour that are characteristic of human social life, but in terms that derive from, and are open to being contradicted by, observation and related empirical evidence.

Claim (iii) amounts to the claim that the sense of participation offered to explain the moral status of people with PMLD is too weak: if what is required is merely some capacity for reciprocity or the ability to wave a stick, then this is also a possibility for high functioning non-human animals. McMahan asserts, of 'radically impaired human beings' that whilst they are capable of 'certain minimal forms of participation' so too are highly developed animals, who 'often participate more extensively than is possible for human beings with lower psychological capacities' (McMahan 2005: 363). For some animals, 'the participation is quite intimate, involving strong reciprocal emotional bonds' (ibid: 364 ), and there is persuasive evidence of animals performing significant functions in human society - as with guide dogs, who remain loyal to and protective of their owners (ibid: $364-5$ ).

Is there a sense of participation that neither demands more than can be expected of most people with PMLD, nor only so much that it could apply to many animals 
besides human beings? The range of participative acts open to a person depends on their physical and psychological capacities, and it is implausible to suppose that all people with PMLD possess more developed capacities of these kinds as compared with all other animals. We are constantly learning more about what people with PMLD are capable of, or would be capable of with suitable human and technological assistance; but equally we are also learning more about the capabilities of higher functioning non-human animals, including dogs, dolphins and great apes (Singer 2011). In so far as participation is conceived in terms of the physical and psychological attributes of individual participants, it is untenable to insist that there is, or that there must be a class of participative acts that fall within the domain of actions available to most people with PMLD but not to any of the non-human animals.

But we need not accept that participation is to be conceived exclusively in terms of the physical and psychological attributes intrinsic to individual participants. There is a distinction between giving an account of the capacities necessary for participation, and an account of the act of participation itself. A child may act out the nursery rhyme 'Heads, Shoulders, Knees and Toes', touching the appropriate part of his body as instructed by his teacher, and smiling or frowning as he is either encouraged or admonished. Whilst not exercising any cognitive capacities exclusive to humans, the rhyme he enacts in consort with his teacher will include social and emotional engagement characteristic of human social intercourse; since, for the exercise to be a success, the teacher is required to display patience and sympathetic understanding, and the child will need to be at ease in her presence, trusting her to be in close proximity to him.

Perhaps the social and emotional capacities referred to here are not unique to human beings; non-human animals can show patience and develop trusting relationships with humans. What is also required, therefore, is a claim about how these capacities are acquired and exercised under human culture, including the norms and practices that distinguish this culture from any other. 'Heads, Shoulders, Knees and Toes' is a product of a local human culture, and the child who participates in this game is doing something that a chimp cannot do, although the chimp may be the higher functioning of the two. 
How should we make sense of this last claim? Human beings have distinctive physical features and most people with PMLD have physical features similar to those of people who do not have any learning disabilities. Some profoundly disabled people have syndromes that lead to the development of physical abnormalities people with Down Syndrome, for example, tend to have reduced muscle tone, a small nose and mouth, broad hands with short fingers, and so on. But in these and other cases, there is no doubt that the body is a human body and that what we see is the body of a human being (which is not to say that what we see is a human being). Human embodiment is bound up with an expressive repertoire that is both made possible and constrained by such physical features as our faces, sensory organs, vocal chords and so on. Humans respond to other humans as having recognisably human features and expressions, and those (other) humans are disposed to reciprocate, or they will learn to do so, to the extent that their bodies and cognitive abilities will allow. Who else besides human beings would laugh out loud on seeing a clown's trousers fall down, break down in floods of tears after being scolded, or chuckle merrily in response to a familiar tease?

Human embodiment is a condition of human culture. Even basic human expressions and gestures are typically learned in a context that includes elaborate local acculturation: reaching out to touch another's hand is in one place an act of kindness, another an affront; the expression of grief in one place will take the form of silent mourning, in another, collective weeping and wailing. We do not respond to the expressions of human beings as such, but to the culturally inflected expressions of human beings. The argument is not, then, that human beings participate in distinctive human practices in virtue of a basic set of common expressions; but that they participate in distinctive practices in virtue of a basic set of expressions learned under a human culture.

Culture conditions even the basic activities of defecation, eating and sexual behaviour. Toileting a child with PMLD requires giving thought to their privacy and dignity; food is typically eaten whilst sitting at a table, and even when it is not it is the norm to use cutlery in the process of feeding someone. The capacities of people with PMLD will themselves have a pervasive influence on norms of conduct and patterns of social interaction. And, generally, homes and schools for children with PMLD 
develop distinctive social environments as intricate and elaborate as in many other complex human organisations.

There are exceptions to the arguments I have offered: not every person with PMLD has the psychological properties required for learning culturally inflected behaviour, and with sufficient assistance from human beings, some non-human animals might become socialised in ways that had been thought of as unique to human beings. These exceptions cannot all be accommodated by the argument presented here, but the damage done is less than it might appear.

One way of countering these last examples is to suggest that a person becomes a member of a human community when and because she is regarded as such by others. Her status as someone who shares a common life with us derives from our recognising her as 'our fellow creature' (Mulhall 2002; Gaita 2000), or from our imaginative sense of her having and leading a human life (Diamond: 1987), or from the narratives we compose, as when we choose to "hold a lissencephalic infant "in personhood"' (Nelson 2002).

These proposals each deserve discussion, but they have in common a view that the moral status of people with PMLD is bestowed or conferred on them by the practices of other people, and, as such, they are all vulnerable to a familiar criticism. Our knowledge of the history of the treatment of people with disabilities demonstrates that there existed, and still exist, common practices, values, narratives and imaginative acts that reveal a conception of disabled people, not as cherished members of a human community, but as defective 'others' who are unwanted, unworthy and less than fully human. If moral status is justified by appeal to common practice then it is hard to see on what basis any practice is to be appraised in terms that extend beyond the practice itself. And if treating or imagining people with PMLD as sub-human and fit for slaughter is common practice, then, where this is common practice, their status is as sub-humans fit for slaughter.

This view cannot be right: practices are not self-authenticating. We require some means of gaining a critical purchase on our practices, and that requires looking outside the practices themselves. The aim is to make some sense of participatory 
capacity that rests neither only on what a person is now capable of, nor on whatever we choose to imagine he is capable of, but on a judgement of what he might be capable of - a judgement that admits of independent assessment in terms that can be recognised and appraised by persons not already implicated in the practices under investigation.

\section{TREATING PEOPLE AS IF THEY HAVE THE POTENTIAL TO PARTICIPATE}

There are two reasons for why we are justified in treating some people as if they have the potential to participate when that potential is in doubt. First, there is a large risk to people who are excluded or marginalised from the rest of their society that they will become vulnerable to abuse or neglect, or that they will be stigmatised, or that they may be denied their lawful rights. The effects of being treated as an outsider, or as sub-human, or as a non-citizen, are often disastrous and lifelong.

In this case, there is a reason to include a person as belonging to a class deserving of a status that might not apply if we considered her case alone. Here, we are appealing to reasoning characteristic of rule consequentialism; the status depends not on a judgement about any one particular case, but about the class of which the case is an example, and we look to the likely consequences of insisting upon one status (exclusion) for that class, rather than another (inclusion).

It might then look as if a justification of this kind implicitly concedes that the person in question lacks potential; for it is necessary to appeal, not to her case, but to others, in order for the justification to get off the ground. But neither the person, nor her potential, drops out of the picture. Policy is often a clumsy instrument for recognising distinctions between persons: there is a reason for caution before endorsing a policy which is likely to be insensitive to fine differences, and so liable to exclude some people whose potential would warrant inclusion if considered on their own merits. And exclusion can have social consequences that are not only very bad, but leave the excluded much worse off than their capacities warrant. A person may lack the potential to participate, but if as a result she is treated as a second class citizen, or as less than fully human, the cumulative effect of denigration may serve to undermine whatever potential she does possess. 
This is connected to a second argument. If we treat people with profound disabilities as if they have the potential to participate, we, and they, are liable to make more effort than otherwise to develop their capacities; equally, if we treat people as if they lack all such potential, we lose an incentive to make every effort to encourage them to realise whatever potential they have (cf Nussbaum 2008: 363-4). Certainly, there is a tendency to underestimate the potential of people with learning difficulties, owing to ignorance, prejudice or a disinclination to meet the cost of care, as when the prejudice that children with Down syndrome were ineducable prevented an accurate appraisal of what they were capable of achieving. This is one reason why Nussbaum proposes that we work as hard on behalf of all people with disabilities to develop their capacities as we would for any other group in society (2006: 190).

This strategy is not shown to be unjustified merely because we can identify some people whose potential to participate is doubtful. There may be a good reason to proceed as if all members of this group are capable of participation just because, although some people may not show signs of potential now, they may yet do so, and nothing less than unqualified support will suffice to overcome the obstacles, including discrimination and prejudice, that now stand in their way. Perhaps only a few of those showing no sign of potential will later surprise us. Still, it may be impossible to determine in advance which people these will be and it remains prudent, therefore, to proceed as if it could be anyone in their peer group.

There is a point here about motivation. The orientation to believe the best about someone may stem from a sunny outlook or a basic conviction that children with PMLD can and will do what is hoped of them. These are intrinsically valuable dispositions. Moreover, someone with a caring or pedagogic role may only be able to carry on as she does if she remains sanguine about her own efficacy and about her children's potential; (too much) sensitivity to recalcitrant cases could have an undermining or destabilising effect. Implacable optimism is a precious quality in people who parent, teach and care for others, and in some cases it is important that disappointment is shrugged off and set-backs not dwelt upon. A certain bullishness may help explain a preference for treating people as if they have a capacity they do not yet have, and the justification for such treatment includes both the intrinsic value 
of an encouraging and optimistic personality, and the effects on the morale and capacity of recipients.

Treating someone as if she has a capability that she is unlikely to acquire, or will never acquire, need not involve any self-deception. We might treat someone this way knowing full well what we are doing, as an expression of a fundamental attitude towards other people, or towards those who are one's children, or pupils, or members of one's local community. The attitude might take the form of always wanting to believe that there is 'more there', that one should 'never say never', that 'anyone is educable. ${ }^{7}$ A person may rationally both abide by the principle that one should 'never say never' and recognise that there may be exceptional cases in which nothing more is to be expected from someone. In this case the principle functions as an expression of a fundamentally sanguine orientation towards other people, rather than as a belief that admits of no exceptions.

Still, even here, there must be some evidential warrant for the belief that a child has the potential to participate: sanguinity cannot alone justify the ascription of potential, for a person might want to ascribe a similar potential to their pet snail. We would then be back with the view that status is acquired merely because it is bestowed.

To avoid this we can appeal to an orientation that is both forward- and backwardlooking. The familiar claim, that a person is more likely to realise their potential in the future if we treat them as if they have this potential now, is part of a forward looking argument. It is related to the claim that 'treating fellow human beings as capable of joining our moral community makes it more likely that they will be able to do so' (Wasserman et al 2013: 13). There is also a backward looking argument, from induction. An experienced teacher may believe that, with sufficient support and encouragement, most of her pupils will eventually learn to take a few steps by themselves, and this belief may be part accounted for, and justified by, the fact that in the past almost all of her children have managed to do this much. It is often impossible to predict which members of a group will manage to make the desired

\footnotetext{
${ }^{7}$ These views are common amongst teachers in special schools for children with profound learning difficulties (BLANK).
} 
progress, and, since most will, it is justifiable to proceed on the assumption that all will. That way, more people are likely to progress than otherwise.

At the same time there are people about whom it is pointless or harmful to believe that they have some potential which the evidence suggests they lack. It would be quite wrong to encourage a child to walk unassisted if the evidence confirms that she can do no such thing, and her condition is anyway deteriorating owing to a life limiting illness. The belief that someone has some potential cannot justifiably be sustained irrespective of the evidence; it can be positively dangerous to treat someone on the basis of beliefs we project onto him, particularly if these stem from thinking that is not only wishful but deluded, and liable to cause harm.

There is a distinction between a belief for which we lack any or much evidence, and a belief which is contradicted by the evidence. And beliefs which may turn out to be false expose us to varying degrees of risk and harm. If negligible harm is caused by treating someone as if he will one day learn to brush his teeth, we might be justified in treating him as if he has this potential unless and until the belief is firmly contradicted. If, however, there is a risk of a bad fall if we treat a child as if he can walk, and there is no evidence to suggest that he will ever manage this, we are unlikely to be justified in treating him in this way.

There remains a wide range of cases in which we are justified in treating people as if they are able to realise potential which may never be realised, or which they may not possess. The justification rests on the good effects of being treated in this way: in virtue of the treatment itself, which might include behaviours that are encouraging and affirmative, rather than discouraging and excluding; in virtue of the positive effects of developing capacities which might otherwise have remained dormant; and in virtue of the social and political status people acquire when treated as capable, or as having the potential of becoming capable, rather than as beyond help and ineducable. 


\section{PARTICIPATION AND WHAT WE OWE TO PEOPLE WITH PMLD}

I have explored the idea that people with PMLD are participants in a common life, or are potential participants in that life, and I have glanced at how this is related to what we owe to them. A few people, certainly, cannot participate in any of the senses I have discussed; and some animals, quite possibly, can participate in some sense of this word, or would be able to participate with sufficient human assistance. If, therefore, there is a moral status that attaches to all and only human beings, participation is neither necessary nor sufficient for this status; 'participation' can only serve as one element in a larger account. There are, nevertheless, two reasons why its place in that account is significant. The first is substantive: the idea of 'participation' draws attention to the social nature of human beings, to the importance of taking part in social interaction, as a means of expressing and developing oneself, and as a source of value that resides in engaging with other people, including the fact of being recognised as a person in one's own right. These points apply to almost all people with profound learning disabilities. The second reason is methodological: any enquiry into participation, as something that helps to explain what is owed to human beings, requires that we look not only at actual capacity, but also at potential capacity and at whether we have reasons to treat people as if they have potential even when that remains a matter of (severe) doubt.

The effort made to encourage people with PMLD to participate suggests that they are worth it. But do we owe it to them to help them? We might argue: people with PMLD need help to participate in valuable social activity; we can help them to participate; therefore, we ought to help. One of several objections here is that what is doing the work in this summary argument to justify assistance is not the value of participation as such, but the value of the person we are enabling to participate. If we owe it to a person to help them join in with our activities it is because she is worth it, as a human being, rather than for any worth that attaches to the act of participation or to her status as a participant.

The case for participation can come to rely on claims about (respect for) personhood or humanity, and, to the extent that it does, we should explain how it is our status as persons, or as members of the human species, that justifies any moral claim that 
human beings make on others. But participation is not to be omitted in a statement of the argument. It is misleading to say that the reason why we owe it to someone to help them join in with us is that they are a human being rather than that they are thereby enabled to participate. For their capacity to participate, and their potential to develop this capacity, is part of what it is to be a human being: human beings are social creatures and it is in their nature to engage in social practices, whether sharing in a joke, eating together or forming relationships. If we owe it to someone to help her join in with us it is not simply because she is a human being, and we owe it to human beings to facilitate their participation; rather, we owe it to someone as a human being whose nature it is to participate in social activity to help them to do that. If, therefore, we want to insist that what we owe to others we owe to them as human beings, then the status of 'being human', as a premise in the argument, is not prior to but includes the capacity for participation. So we owe it to a child with PMLD to help her laugh and join in playful activity not simply because she is a human being but because, being human, it is in her nature to laugh and participate in playful activity.

Not all acts of participation are eligible for assistance; some are trivial, others reprehensible. It may be in our nature to join in with others' gossiping or with acts of aggression, but there is typically no reason why we should be assisted in doing so. We will have, therefore, to identify examples of participation that are intrinsically valuable and which, where support is necessary, impose demands on people who are in a position to provide it. So it has been argued that people should receive an education in order that they are enabled to participate in political decision making, because participation in political decision making is intrinsically valuable or a matter of basic human rights. Similarly, we might claim that people with PMLD should receive the education and social support they need in order that they can participate in social life, because participation in social life is intrinsically valuable and constitutes a basic social good in their lives. This, at any rate, is an example of an argument that might be developed to show how participation on the part of people with PMLD is related to what they are owed.

Perhaps any appeal to participation of the kind I have considered is simply to betray a fundamental misunderstanding about why we value human beings. Mulhall appears to think so: it is enough, on his view, that anyone, irrespective of ability, is 
our fellow human being, vulnerable to the 'shocks and ills to which all human flesh and blood is heir' (Mulhall 2002: 18). This is a powerful thought; and it is a hopeful thought for those human beings who could not be conceived as participants in any of the senses explored in this paper. But if what Mulhall is appealing to really is enough, we will have to explain what it is about being human, as such, and independently of an appeal to participation or any other capacity, which justifies our treating someone as 'fully' human and as our moral equal. And that will require an account of the nature and status of human beings as fraught with difficulty as anything offered here. 


\section{REFERENCES}

American Psychiatric Association. 2000. Diagnostic and Statistical Manual of Mental Disorders, Fourth Edition (DSM-IV-TR). Arlington, VA: American Psychiatric Association.

American Psychiatric Association. 2013. Diagnostic and Statistical Manual of Mental Disorders. Fifth Edition. Arlington, VA: American Psychiatric Association.

Dee, Leslie., et al. 2002. Enhancing Quality of Life: A Literature Review. Cambridge: Skill and University of Cambridge.

Diamond, Cora. 1978. Eating Meat and Eating People. Philosophy 53, 206: 465-479.

DiSilvestro, Russell. 2010. Human Capacities and Moral Status. Dordrecht: Springer.

Gaita, Raymond. 2000. Goodness beyond Virtue. In A Common Humanity, $\left(2^{\text {nd }}\right.$ Edition), Raymond Gaita, 17-27. London: Routledge.

Graham, Keith. 2002. Practical Reasoning in a Social World. Cambridge: Cambridge University Press.

Gunnarson, Logi. 2008. Great Apes and the Severely Disabled Moral Status and Thick Evaluative Concepts. Ethical Theory and Moral Practice 11: 305-326.

Kittay, Eva., 2005. At the Margins of Personhood. Ethics 116: 100-131

Hilde Lindemann Nelson. 2002. What Child Is This? Hastings Center Report 32, 6: 29-38.

McMahan, Jeff. 2005. Our Fellow Creatures. The Journal of Ethics 9: 353-380.

Mulhall, Stephen. 2002. Fearful Thoughts. London Review of Books 24, 16: 18. 
Njaji, Francis, and Peter Tymms. 2009. The P scales: Assessing the Progress of Children with Special Educational Needs. Chichester: Wiley-Blackwell.

Nussbaum, Martha. 2006, Frontiers of Justice: Disability, Nationality and Species Membership. Harvard: Harvard University Press.

Ofsted. 2013. Profound Education (formerly St Margaret's School). Ofsted. file:///C:/Users/owner/Downloads/The Children s Trust School Ofsted social care inspection report October 2013.pdf. Accessed 4 February 2015.

Qualifications and Curriculum Authority. 2009. The P Scales. London: Qualifications and Curriculum Authority.

Scanlon, Tim. 2000. What We Owe to Each Other. MA: Harvard University Press.

Schuitema, Karin, Gill Brigg, and BLANK. Forthcoming. Children with profound and multiple learning difficulties: laughter, capability and relating to others.

Silvers, Anita, and Francis, Leslie. 2009. Thinking about the good: reconfiguring liberal metaphysics (or not) for people with cognitive disabilities. Metaphilosophy 40 (3/4): 475-498.

Singer, Peter. 2011. The Expanding Circle. Princeton: Princeton University Press.

BLANK.

Wasserman, David, and Jeff McMahan. 2012. Cognitive Surrogacy, Assisted Participation, and Moral Status. In Medicine and Social Justice (2 ${ }^{\text {nd }}$ Ed.), ed. Rosamond Rhodes, Margaret Battin, and Anita Silvers, 325-333. Oxford: Oxford University Press. 
Wasserman, David, Adrienne Asch, Jeffrey Blustein and Daniel Putnam. 2013. Cognitive Disability and Moral Status'. The Stanford Encyclopedia of Philosophy, Edward N.

Zalta (ed.).

$\mathrm{URL}=<\mathrm{http}: / /$ plato.stanford.edu/archives/fall2013/entries/cognitive-disability/>. Accessed 17 January 2015. 